Noname manuscript No.

(will be inserted by the editor)

\title{
A generalized BER prediction method for differential chaos shift keying system through different communication channels
}

\author{
Georges Kaddoum • François Gagnon • \\ Pascal Chargé · Daniel Roviras
}

Received: date / Accepted: date

\begin{abstract}
In this paper, we present a generalized accurate methodology to predict the bit error rate performance for non-coherent chaos-based communication systems. The Gaussian approximation approach, which is widely used to compute the performance of such systems, leads to inaccurate results, especially with respect to low spreading factors. Our new approach based on the chaos bit energy distribution gives accurate results even for low spreading factors. The system is studied and simulated under an additive white Gaussian noise, Rice and Rayleigh channels. Finally, we compare our approach to the Gaussian approximation approach. Computer simulations shows a high accuracy for our method, especially for small spreading factors.
\end{abstract}

Keywords DCSK system; energy distribution; bit error rate; communication channels.

G. Kaddoum

LACIME Laboratory, École de technologie supérieure, 1100 Notre-Dame Street West, Montreal (Quebec) H3C 1K3 Canada

Tel.: (514) 396-8997

Fax: (514) 396-8684

E-mail: georges.kaddoum@lacime.etsmtl.ca

F. Gagnon

LACIME Laboratory, École de technologie supérieure, 1100 Notre-Dame Street West, Montreal (Quebec) H3C 1K3 Canada

Tel.: (514) 396-8997

Fax: (514) 396-8684

E-mail: francois.gagnon@etsmtl.ca

P. Chargé

LATTIS Laboratory, University of Toulouse, 135 Avenue de Rangueil, 31077 Toulouse cedex

4, France

E-mail: pascal.charge@insa-toulouse.fr

D. Roviras

LAETITIA Laboratory, CNAM Paris, 292 rue Saint-Martin,75003 Paris, France

E-mail: daniel.roviras@cnam.fr 


\section{INTRODUCTION}

Over the past decade, many different chaos-based communication systems have been proposed, and interest in them can be attributed to the advantages provided by chaotic signals, such as robustness in multipath environments, and resistance to jamming and interception [7],[10]. Among the various digital chaos-based communication schemes, coherent chaos-shift-keying (CSK) [10], chaos-based DS-CDMA [7], and non-coherent Differential Chaos Shift Keying (DCSK) [9] have been widely studied and evaluated. One of the drawbacks of coherent chaos-based communication systems such as the CSK is that they involve synchronization. Since the chaos synchronization proposed by Pecora and Carroll in [14] is still practically impossible to achieve in a noisy environment, the coherent system cannot be used in realistic application. Furthermore, the DCSK system represents a robust non-coherent scheme in which an exact knowledge of the chaotic signal at the receiver side is not required. Because of its simple structure, the DSCK system is one of the most promising chaos-based communication schemes in terms of a feasible implementation, and both the theory and the implementation of the system have indeed undergone significant research [8], [6],[13].

In the DCSK system, the bit duration is divided into two equal slots, the first being is the chaotic reference signal. Depending on the bit to be sent, the reference signal is either repeated if the bit to send is +1 , or multiplied by a factor of -1 in the second case and transmitted in the second slot. At the receiver, the signal is delayed by a duration of half a bit and correlated with an undelayed signal to estimate the transmitted bit information.

The advantage of the DCSK over the CSK is that with the DCSK, the threshold level at the receiver is always set to zero, and is independent of the noise level [10]. However, the bit frequency can easily be determined from the transmitted signal. The result however, is a reduction in the security of the system. To overcome this security problem, Francis et al have proposed in [11] a method called the permutation-based $D C S K$ or $P-D C S K$, aimed at eliminating this similarity between the reference slot and the carrier information slot.

The performance of single and multi-user DCSK systems over an additive white Gaussian noise (AWGN) channel has been widely studied over the past decade [18],[15]. The performance of a frequency-modulated DCSK (FM-DCSK) communication system over a multipath fading channel was simulated in [6],[17] The performance of a conventional single-user DCSK system over a multipath fading channel was investigated in [20] and later extended in [16] to a multipath fading channel with delay spread.

Many approaches have been considered for computing the BER performances of the DCSK system, with the first widely used one being the Gaussian approximation (GA). This approximation considers that the correlator output follows the normal distribution. Applied to the DCSK system over an AWGN or multipath channel in [15],[19], this method provides rather good estimates of the BER for very large spreading factors, but when the spreading factor is small, the results produced by the Gaussian approximation method are rather disappointing.

In [20], the computation methodology developed in [12],[18] extended to compute the BER performance of the single-user DCSK over an m-distributed fading channel. The Gaussian assumption is not used to derive the BER expression, and their approach enables the dynamics properties of the chaotic sequence by integrating the BER expression for a given chaotic map over all possible chaotic sequences for a given spreading factor. This latter method is compared to the BER computation under the Gaussian 
assumption in [20], and seems more realistic to match the exact BER. However, as indicated in [20], the drawback of the proposed method is the high calculation difficulty it poses. Note that all approaches proposed in the literature use the numerical integration to compute the BER expression of the DCSK system over an m-distributed fading channel.

Since approaches that have been previously presented so far are either not valid for small spreading factors or involve highly complex computations, we we develop a simple and accurate method in this paper for computing the exact BER performance for a single-user DCSK system. The system is evaluated over an AWGN and over a $\mathrm{m}$-distributed fading channels. Our proposed method mainly proposes the computation of the probability density function (PDF) of the chaotic bit energy and the integration of the BER over all possible values of the PDF. The novelty of this paper lies in the fact that it gives an exact BER expression without neglecting the dynamical properties of chaotic sequences with low computing charges.

The paper is organized as follows. Section 2 explains the DCSK system. The Gaussian approximation and our proposed methodology for computing the BER are given in Section 3. Numerical results are presented in section 4, and the paper is concluded in Section 5.

\section{DCSK system}

\subsection{Chaotic generator}

The proposed approach is valid for any type of chaotic sequence. In this paper, the Chebyshev polynomial function $(\mathrm{CPF})$ of order 2 has been chosen.

$$
x_{k}=2 x_{k-1}^{2}-1
$$

\subsection{Transmitter structure}

The structure of the DCSK system is illustrated in Figure 1, and the modulation process is shown in Figure 1(a), where each bit $s_{i}=\{+1,-1\}$ is represented by two sets of chaotic signal samples, the first set of which represents the reference, while the second carries the data. Let $2 \beta$ be the spreading factor, defined as the number of chaotic samples sent for each bit, where $\beta$ is an integer. The emitted DCSK signal is:

$$
e_{k}= \begin{cases}x_{k} & k=1 \ldots \beta \\ s_{i} x_{k-\beta} & k=\beta+1 \ldots 2 \beta\end{cases}
$$

\subsection{Communication channels}

The channel model is given by:

$$
\alpha=\sqrt{2 K}+a+j b
$$




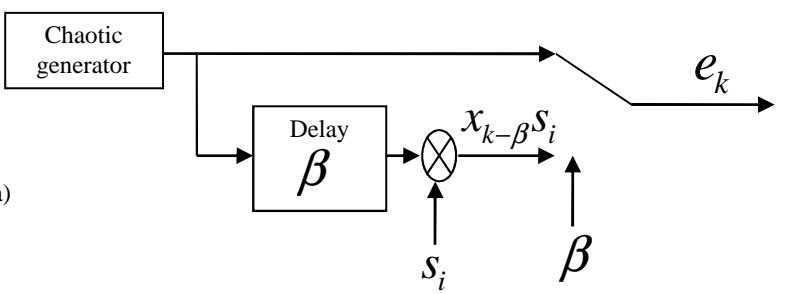

(b)
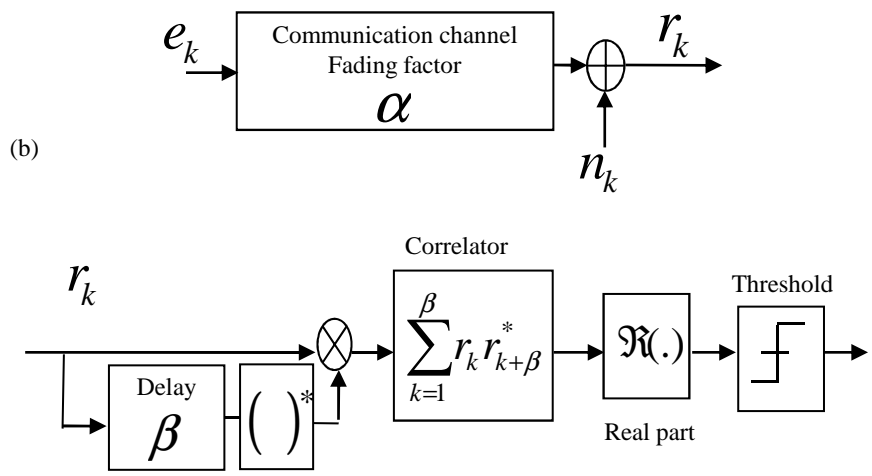

(c)

Fig. 1 (a) Transmitter structure, (b) channel model, (c) Receiver structure

where $K$ is a deterministic variable, known as the Rice factor, and $a, b$ are two independent Gaussian random variables with zero mean and variances equal to 1 . $K$ can be seen as a shape parameter, since it fixes the nature of the channel. When $K^{(m)}=0$, it is a Rayleigh channel (i.e. $|\alpha|$ is distributed according to a Rayleigh distribution); when $K$ tends to infinity, it is a Gaussian channel (i.e., $|\alpha|$ tends to a constant variable); for intermediate values of $K$, it is a Rice channel (i.e., $|\alpha|$ is distributed according to a Rice distribution). An additive complex circular white Gaussian noise $n_{k}$ is added at the output of the channel, with a two-side power spectral density equal to $N_{0}$. The received signal is:

$$
r_{k}=\alpha e_{k}+n_{k}
$$

In this paper, the channel coefficient $\alpha$ is kept constant during the bit duration and for an AWGN channel, the channel coefficient is constant $\alpha=1$. 


\subsection{Receiver structure}

The block diagram of the DCSK receiver is shown in Figure 1 (c). The received signal $r_{k}$ passes by a correlator, where the reference samples and the corresponding data samples are correlated. The decision variable at the output of the correlator for a given bit $i$ is then:

$$
\begin{gathered}
D_{i}=\Re\left(\sum_{k=1}^{\beta} r_{k} r_{k+\beta}^{*}\right) \\
=\Re\left(\sum_{k=1}^{\beta}\left(\alpha_{i} x_{k}+n_{k}\right)\left(\alpha_{i}^{*} s_{i} x_{k}+n_{k+\beta}^{*}\right)\right) \\
=A+B+C \\
A=\sum_{k=1}^{\beta} s_{i} \alpha_{i}^{2} x_{k}^{2}, \\
B=\Re\left(\sum_{k=1}^{\beta} \alpha x_{k} n_{k+\beta}^{*}+\alpha^{*} s_{i} x_{k} n_{k}\right),
\end{gathered}
$$

and

$$
C=\Re\left(\sum_{k=1}^{\beta} n_{k} n_{k+\beta}^{*}\right)
$$

where $R($.$) , and *$ are the real and the complex conjugate operators, respectively.

The decoded bit is then related to the sign of the decision variable.

$$
\hat{s}_{i}= \begin{cases}+1, & \text { if } D_{i}>0 \\ -1, & \text { if } D_{i}<0\end{cases}
$$

\section{Performance of the DCSK system}

3.1 BER expression under Gaussian approach

In this section, we briefly present the Gaussian approach, and later we will compare and discuss the results of our proposed method. Based on the Gaussian approximation, the correlator output $D_{i}$ follows a normal distribution. Thanks to the central limit theorem, the statistics of the of decision variable are:

$$
\begin{gathered}
E[A]=\beta s_{i}\left|\alpha_{i}\right|^{2} E\left[x_{k}^{2}\right] \\
E[B]=E[C]=0 \\
\operatorname{Var}[A]=\beta\left|\alpha_{i}\right|^{4} \operatorname{var}\left[x_{k}^{2}\right]
\end{gathered}
$$




$$
\begin{gathered}
\operatorname{Var}[B]=\beta N_{0}\left|\alpha_{i}\right|^{2} \operatorname{var}\left[x_{k}\right] \\
\operatorname{Var}[C]=\beta N_{0}^{2} / 4
\end{gathered}
$$

and

$$
\operatorname{cov}[A, B]=\operatorname{cov}[A, C]=\operatorname{cov}[B, C]=0
$$

where $E[$.$] and \operatorname{var}[$.$] denote the mean and the variance operators, respectively, and$ $\operatorname{cov}[L, M]$ denotes the covariance of $L$ and $M$.

Finally, we have:

$$
\begin{gathered}
E\left[D_{i}\right]=\beta s_{i}\left|\alpha_{i}\right|^{2} E\left[x_{k}^{2}\right] \\
\operatorname{var}\left[D_{i}\right]=\beta\left|\alpha_{i}\right|^{4} \operatorname{var}\left[x_{k}^{2}\right]+\beta N_{0}\left|\alpha_{i}\right|^{2} \operatorname{var}\left[x_{k}\right]+\beta N_{0}^{2} / 4
\end{gathered}
$$

The decision variable at the output of the correlator is approximated by random a Gaussian variable. Using equations (6), (7), the bit error probability is:

$$
\begin{aligned}
& B E R=\frac{1}{2} \operatorname{Pr}\left(D_{i}<0 \mid s_{i}=+1\right)+\frac{1}{2} \operatorname{Pr}\left(D_{i}>0 \mid s_{i}=-1\right) \\
& \quad=\frac{1}{2} \operatorname{erfc}\left(\frac{E\left[D_{i} \mid s_{i}=+1\right]}{\sqrt{2 \operatorname{var}\left[D_{i} \mid s_{i}=+1\right]}}\right)
\end{aligned}
$$

where $\operatorname{erfc}(x)$ is the complementary error function defined by:

$$
\operatorname{erfc}(x) \equiv \frac{2}{\sqrt{\pi}} \int_{x}^{\infty} e^{-\mu^{2}} d \mu
$$

Since the chaotic signal is non periodic, equation (8) is an approximated formula of the BER which is not enough to match the exact performance of the DCSK system. Only the low-order moment of the chaotic sequence has been considered. By considering the mean of the decision variable, the term $\beta E\left[x_{k}^{2}\right]$ is independent from the bit $s_{i}$. Equation (9) can only be valid for a very large spreading factor.

$$
E\left[x_{i, 1}^{2}+x_{i, 2}^{2}+\ldots+x_{i, \beta}^{2}\right] \approx E\left[x_{i+1,1+\beta}^{2}+x_{i, 2+\beta}^{2}+\ldots+x_{i, \beta+\beta}^{2}\right] \approx \beta E\left[x_{k}^{2}\right]
$$

Otherwise, equation (9)can not be satisfied for a small spreading factor. In this case, the Gaussian approximation leads to incarcerate results.

In this paper, the CPF map is used. The invariant probability density function of $x$ is given in [1]:

$$
\rho(x)=\left\{\begin{array}{l}
\frac{1}{\pi \sqrt{1-x^{2}}}, \quad \text { if }|x|<1 \\
0, \quad \text { otherwise }
\end{array}\right.
$$

From equation (10), we can compute the mean and the variance of $x^{2}$ by:

$$
E\left[x^{2}\right]=\int_{-\infty}^{\infty} x^{2} \rho(x) d x=\int_{-1}^{1} x^{2} \frac{1}{\pi \sqrt{1-x^{2}}} d x=\frac{1}{2}
$$


and

$$
\operatorname{Var}\left[x^{2}\right]=E\left[x^{4}\right]-\left(E\left[x^{2}\right]\right)=\frac{1}{8}
$$

By replacing (11), (12) into equation (8), the BER of the CPF map becomes

$$
B E R_{G A}=\frac{1}{2} \operatorname{erfc}\left(\left(\frac{1}{N}+\frac{4}{\gamma_{G A}}+\frac{2 \beta}{\gamma_{G A}^{2}}\right)^{-1 / 2}\right)
$$

where $\gamma_{G A}=|\alpha|^{2} E_{b} / N_{0}$ is the signal to noise ration per symbol, and $E_{b}=2 \beta E\left[x^{2}\right]$ is the bit energy.

For an AWGN channel, the fading factor is constant (i.e., $\alpha=1$ ), the BER of the DCSK system under a Gaussian approximation for an AWGN channel is given by equation (13) with $\gamma_{G A}=E_{b} / N_{0}$.

For an m-distributed fading channel, the mean BER of the DCSK system is obtained by integrating (13) over all possible values of the channel gain:

$$
B E R_{G A}^{\text {fading }}=\int_{0}^{\infty} B E R_{G A}\left(\gamma_{G A}\right) f_{\text {fading }}\left(\gamma_{G A}\right) d \gamma_{G A}
$$

Depending on the variable $\alpha$, the function $f_{\text {fading }}($.$) can be the probability density$ function of a Rice or a Rayleigh distribution.

\subsection{Performance analysis under energy distribution approach}

In this section, we present a new approach for computing the BER of the DCSK system. This approach is based on the bit energy distribution of the spreading chaotic sequence multiplied by the channel coefficient. The methodology results in to an accurate performance prediction for any spreading factor with a low computing charge. For a given fixed bit $s_{i}$, the received bit energy $E_{b c}^{(i)}$ is constant, and the mean and variance of the decision variable are derived as follows:

$$
\begin{gathered}
E[A]=s_{i}\left|\alpha_{i}\right|^{2} E\left[\sum_{k=1}^{\beta} x_{k}^{2}\right] \\
E[A]=s_{i}\left|\alpha_{i}\right|^{2} \frac{E_{b c}^{(i)}}{2}
\end{gathered}
$$

where $E_{b c}^{(i)}$ is the chaotic bit energy

Since the noise samples are uncorrelated, the means of the variables $B$ and $C$ are:

$$
E[B]=E[C]=0
$$

Finally the mean of the decision variable is:

$$
E\left[D_{i}\right]=s_{i}\left|\alpha_{i}\right|^{2} \frac{E_{b c}^{(i)}}{2}
$$

Expression (15) relies on the fact that $A$ is the useful signal, $B$ and $C$ are zero mean random quantities. 
For a constant received bit energy $E_{b c}^{(i)}$, the variances are computed as follow:

$$
\begin{gathered}
\operatorname{Var}[A]=E\left[\left(s_{i}\left|\alpha_{i}\right|^{2} \frac{E_{b c}^{(i)}}{2}\right)^{2}\right]-\left(s_{i}\left|\alpha_{i}\right|^{2} \frac{E_{b c}^{(i)}}{2}\right)^{2}=0 \\
\operatorname{Var}[B]=\left|\alpha_{i}\right|^{2} \frac{E_{b c}^{(i)}}{2} N_{0} \\
\operatorname{Var}[C]=\beta N_{0}^{2} / 4
\end{gathered}
$$

and

$$
\operatorname{cov}\{A, B\}=\operatorname{cov}\{A, C\}=\operatorname{cov}\{B, C\}=0
$$

Since the three terms of (5) are uncorrelated, the conditional variance of the decision variable for a given bit $s_{i}$ is:

$$
\operatorname{var}\left[D_{i}\right]=\left|\alpha_{i}\right|^{2} \frac{E_{b c}^{(i)}}{2} N_{0}+\beta N_{0}^{2} / 4
$$

In order to compute the BER with our approach, the error probability must first be evaluated for a given received energy. Considering the bit energy (or chaotic chips) as a deterministic variable, the decision variable at the output of the correlator is necessarily a random Gaussian variable, and this error probability becomes:

$$
\begin{aligned}
P_{e r}^{(i)} & =\frac{1}{2} \operatorname{erfc}\left(\frac{E\left[D_{i}\right]}{\sqrt{2 \operatorname{var}\left[D_{i}\right]}}\right) \\
& =\frac{1}{2} \operatorname{erfc}\left(\frac{\alpha_{i}^{2} \frac{E_{b c}^{(i) 2}}{2}}{\sqrt{2\left(\left|\alpha_{i}\right|^{2} \frac{E_{b c}^{(i) 2}}{2} N_{0}+\beta N_{0}^{2} / 4\right)}}\right)
\end{aligned}
$$

To compute the BER performance of the DCSK system, many approaches have considered the transmitted bit energy to be constant [19], [15]. Given the non-periodic nature of chaotic signals, it is clear that the transmitted bit energy after spreading by chaotic sequences varies from one bit to another [3]. The mean BER of the system is then obtained by integrating (17) over all possible values of the bit energy and channel gain:

The mean BER can also be expressed as:

$$
B E R=\int_{0}^{\infty} \frac{1}{2} \operatorname{erfc}\left(\sqrt{\frac{\gamma}{4 N_{0}}\left(1+\frac{\beta N_{0}}{2 \gamma}\right)^{-1}}\right) p(\gamma) d(\gamma)
$$

where $p(\gamma)$ is the probability density function of the variable $\gamma$ and $\gamma=|\alpha|^{2} E_{b c}^{(i)}$ is the received bit energy. 


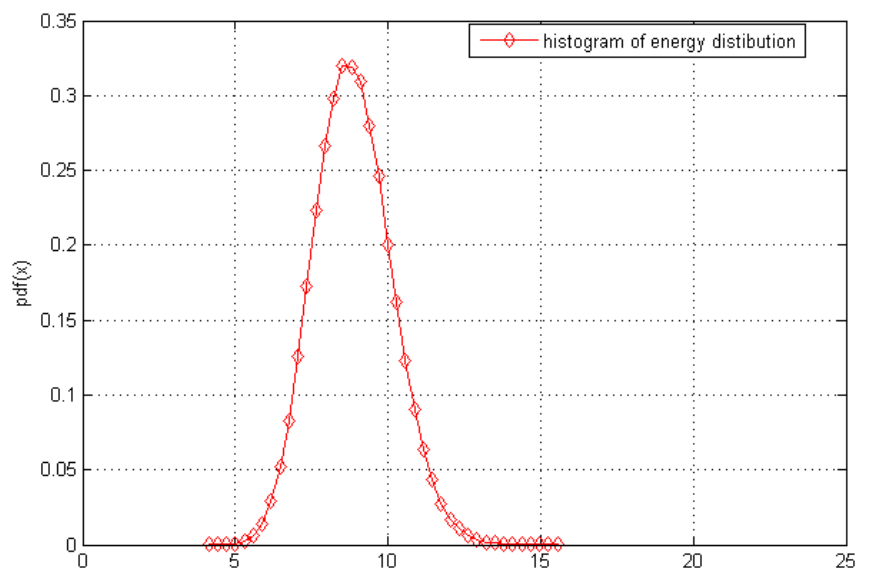

Fig. 2 Histogram of variable $\gamma$ for $\beta=20$ and $K=0 d B$

\subsubsection{BER computation methodology}

Computing (18) requires that we know the PDF of $\gamma$. The PDF of the root square of $\gamma$ is estimated in [4] and results in an analytical BER expression for coherent chaos-based communication systems. In the non-coherent systems case, the analytical expression seems to be intractable because the denominator of the BER contains the term $\gamma$. To overcome this problem, a solution relies on a numerical integration of (18). To compute the BER expression, we have fitted the histogram of the variable for the chaotic sequence under study. Figure 2 shows the histogram of the bit energy of the Chebyshev chaotic function for a delay $\beta=10$ and for a channel gain $K=0 \mathrm{~dB}$.

Using the appropriate histogram for the corresponding channel (i.e., the histogram of Figure 2), we can compute the BER expression of (18) for the communication channel by using the following expression:

$$
B E R \approx \sum_{n=1}^{C} \frac{1}{2} \operatorname{erfc}\left(\sqrt{\frac{\gamma^{(n)}}{4 N_{0}}\left(1+\frac{\beta N_{0}}{2 \gamma^{(n)}}\right)^{-1}}\right) P\left(\gamma^{(n)}\right)
$$

where $C$ is the number of histogram classes and $P\left(\gamma^{(n)}\right)$ is the probability of having the energy within the interval centred in $\gamma^{(n)}$.

Note that all the proposed approaches to computing the performance of the DCSK system over an m-distributed fading channels in the literature need a numerical integration. The first part of these approaches considers the Gaussian approximation which leads to inaccurate results especially when the spreading factor is low [16],[19]; while the second part is an exact derivation of the BER but with a high calculation complexity [20]. Our proposed approach can be applied for any type of chaotic sequence and for any type of communication channel with very simple operations: histogram of the received bit energy (i.e., $\gamma$ ) followed by a numerical integration. Furthermore, this approach explores the dynamics properties of chaotic sequences without any approximation, and yields results with very high degrees of accuracy. Moreover, because of 


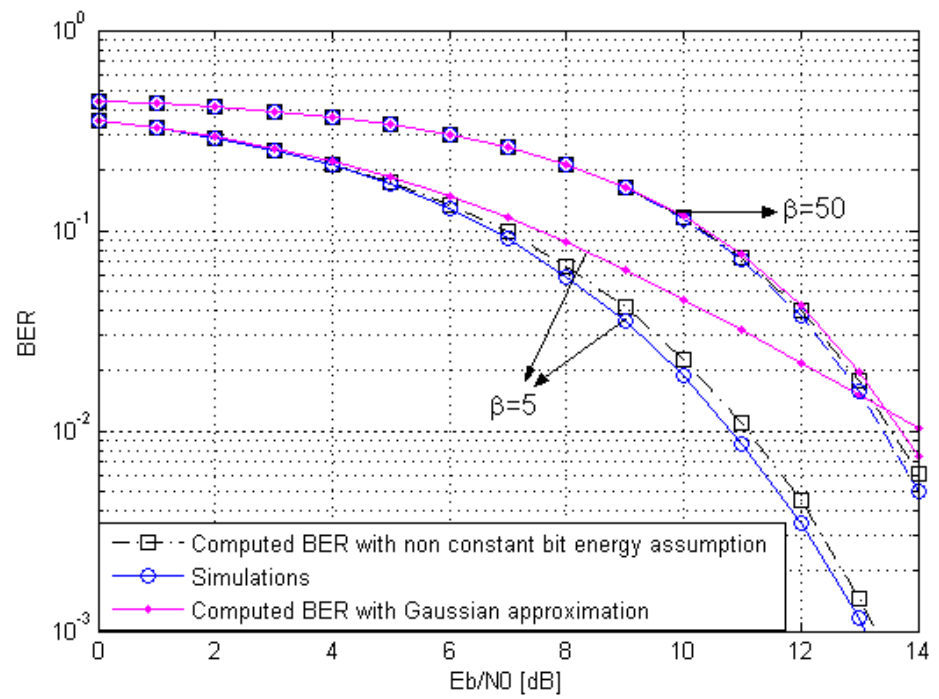

Fig. 3 Exact BER expression, Simulations, Computed BER expression with Gaussian approximation for spreading factors $\beta=5 ; 50$ of CPF map and AWGN channel

its accuracy and computing simplicity, this method will be helpful for engineers or researchers to evaluating and comparing the performance of chaos-based communication systems.

\section{Simulations}

Figure 3 presents the BER curves obtained from our BER of expression (19) based on the bit received bit energy distribution of the chaotic sequence, the Monte Carlo simulations of the DCSK system, and the BER expression based on Gaussian approximation given in (13). It clearly appears that there is an excellent match between simulations and our computed BER expression especially when the spreading factor is low. For low spreading factors we can see that the BER expression based on the Gaussian approximation can not match the Monte Carlo curves when the noise variance decreases. For a high spreading factors, the bit energy variation is small [2],[5],[3], and in this case, the Gaussian approximation can estimate the performance of the DCSK system. The higher is the spreading factor, the smaller the difference between the two performance analysis methods compared. We can also see for a high spreading factor $(\beta=50)$, over an AWGN channel, and for a single user case, the performance of the DCSK system is worse than when the spreading factor is equal to $\beta=5$. One of the reasons of performance degradation is to the variance of the squared noise, which is a function of the spreading factor. The dependence of the performance on the spreading sequence length and the optimal spreading factor is fully discussed in [15].

Figure 4 and 5 present the BER curves obtained from our BER expression given in (19) and from the Monte Carlo simulations of the DCSK system. The perfect match between the simulations and the computed BER expression for any spreading factor proves the accuracy of the proposed approach for any communication channel. 


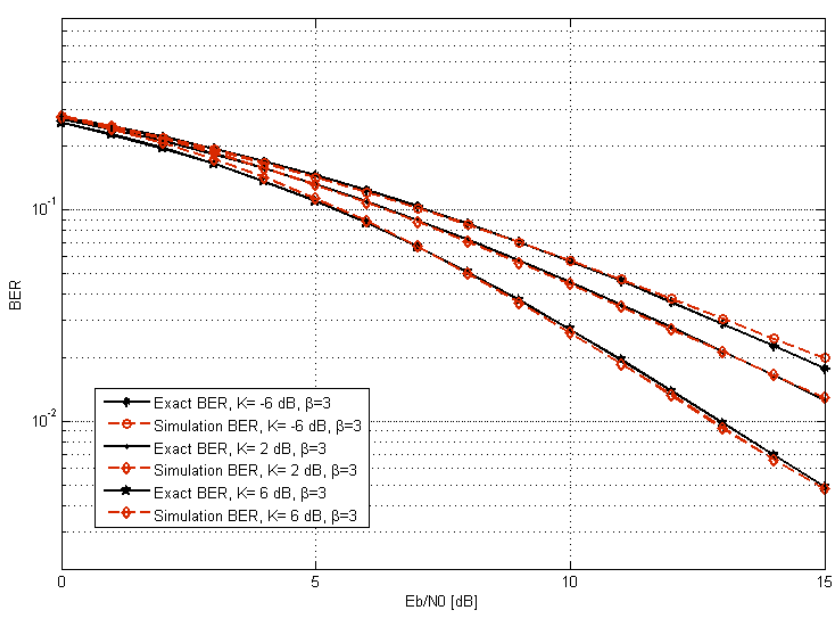

Fig. 4 BER performances of DCSK system for m-distributed fading channels with gains $K=-6 ; 2 ; 6 d B$ and spreading factor $\beta=3$

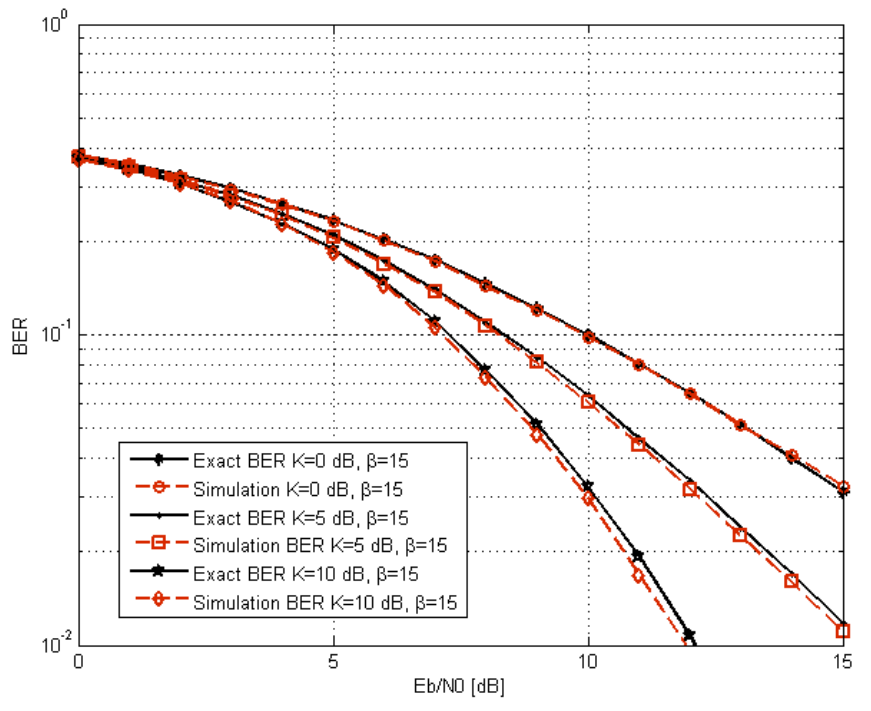

Fig. 5 BER performances of DCSK system for m-distributed fading channels with gains $K=0 ; 5 ; 10 d B$ and spreading factor $\beta=15$

\section{Conclusion}

In this paper, we have proposed a general method for computing the performance of the DCSK system over many communication channels. The computation method is presented and evaluated under an AWGN, a Rice and a Rayleigh channel for a 
single-user case. This latter method can successfully be applied in multi-user case, for any chaotic map, and for any communication channel. Since chaotic sequences are non-periodic, the Gaussian assumption doses not yield very accurate results. The new approach used to derive the BER is based on the received bit energy distribution. The first and the second moments of the decision variable are computed, and the numerical integration of the BER is presented. Simulation results show accurate results and a perfect match with simulations, even when the spreading factor is small. Furthermore, simulation results prove that our approach outperforms the Gaussian approximation in estimating the performance of the DCSK system. This new computation method can be applied for any type of chaotic sequence, with very simple operations: histogram of the transmitted bit energy followed by a numerical integration. Moreover, this approach explores the dynamics properties of chaotic sequences, and yields results having a very high degree of accuracy.

\section{References}

1. Geisel, T., Alouini, M.S.: Statistical properties of chaos in Chebyshev map. Physics Letters A 6, 263-266 (1984)

2. Kaddoum, G., Chargé, P., Roviras, D., Fournier-Prunaret, D.: Comparison of chaotic sequences in a chaos based DS-CDMA system. In: Proc. International symposium on nonlinear theory and its applications. Vancouver, Canada (2007)

3. Kaddoum, G., Chargé, P., Roviras, D., Fournier-Prunaret, D.: A methodology for bit error rate prediction in chaos-based communication systems. Springer,Birkhäuser, Circuits, Systems and Signal Processing DOI: 10.1007/s00034-009-9124-5 (2009)

4. Kaddoum, G., Coulon, M., Roviras, D., Chargé, P.: performance of multi-user asynchronous chaos-based communication systems through m-distributed fading channel. In: Proc. EUSIPCO. Glasgow, Scotland (2009)

5. Kaddoum, G., Roviras, D., Chargé, P., Fournier-Prunaret, D.: Analytical calculation of BER in communication systems using a piecewise linear chaotic map. In: Proc. European Conference on Circuit Theory and Design. Seville, Spain (2007)

6. Kennedy, M.P., Kolumbán, G., Kis, G., Jákó, Z.: Performance evaluation of FM-DCSK modulation in multipath environments. IEEE Trans. Circuits and Systems 47, 1702-1711 (2000)

7. Kennedy, M.P., Rovatti, R., Setti, G.: Chaotic Electronics in Telecommunications. CRC press London (2000)

8. Kolumbán, G., Kis, G., Jákó, Z., Kennedy, M.P.: FM-DCSK: A robust modulation scheme for chaotic communications. Trans. Fundamentals of Electronics Communications and Computer Sciences 89, 1798-1802 (1998)

9. Kolumbán, G., Vizvari, G.K., Schwarz, W., Abel, A.: Differential chaos shift keying : a robust coding for chaos communication. In: Proc. International Workshop on Nonlinear Dynamics of Electronic Systems, pp. 92-97. Seville, Spain (1996)

10. Lau, F.C.M., Tse, C.K.: Chaos-Based Digital Communication Systems. Springer-Verlag (2003)

11. Lau, F.M., Cheong, K.Y., Tse, C.K.: Permutation-based DCSK and multiple-access dcsk systems. IEEE Trans. Circuits and Systems 50, 733-742 (2003)

12. Lawrance, A.J., Ohama, G.: Exact calculation of bit error rates in communication systems with chaotic modulation. IEEE Trans. Circuits and Systems 50, 1391-1400 (2003)

13. Mandal, S., Banerjee, S.: Analysis and CMOS implementation of a chaos-based communication system. IEEE Trans. Circuits and Systems 51, 1708-1722 (2004)

14. Pecora, L.M., Carroll, T.L.: Synchronization in chaotic systems. Phys. Rev. A 64, 821-823 (1990)

15. Sushchik, M., Tsimring, L.S., Volkovskii, A.R.: Performance analysis of correlation-based communication schemes utilizing chaos. IEEE Trans. Circuits and Systems 47, 1684-1691 (2000)

16. Xia, Y., Tse, C.K., Lau, F.C.M.: Performance of differential chaos-shift-keying digital communication systems over a multipath fading channel with delay spread. IEEE Trans. Circuits Syst. II, Express Briefs 51, 680-684 (2004) 
17. Xia, Y., Tse, C.K., Lau, F.C.M., Kolumbn, G.: Performance of frequency-modulated differential chaos-shift-keying communication system over multipath fading channels with delay spread. Int. J. Bifurc. Chaos 15, 40274033 (2005)

18. Yao, J., Lawrance, A.J.: Performance analysis and optimization of multi-user differential chaos-shift keying communication systems. IEEE Trans. Circuits and Systems 53, 2075$2091(2006)$

19. Zhou, Z., Wang, J., Ye, Y.: Performance of multiple-access communication over multipath channel with delay spread. Brikhaüser Trans. Circuits Syst and Sig. Process 2008, 507-518 (2008)

20. Zhou, Z., Wang, J., Ye, Y.: Exact ber analysis of differential chaos shift keying communication system in fading channel. Springer Trans. Wireless Pers Commun 2009, doi 10.1007/s 11,277-009-9685-4 (2009) 